\title{
Investigating Novel and Conventional Biomarkers for Post-resuscitation Prognosis: The Role of Cytokeratin-18, Neuron-specific Enolase, and Lactate
}

\section{Beata Csiszar}

1st Department of Medicine, Division of Cardiology, University of Pecs, Medical School, Pecs, Hungary Almos Nemeth

Anaesthesiology and Intensive Therapy Unit, Uzsoki Hospital, University of Semmelweis, Budapest, Hungary

\section{Zsolt Marton}

1st Department of Medicine, Division of Cardiology, University of Pecs, Medical School, Pecs, Hungary Janos Riba

1st Department of Medicine, Division of Cardiology, University of Pecs, Medical School, Pecs, Hungary

\section{Peter Csecsei}

Department of Neurosurgery, University of Pecs, Medical School, Pecs, Hungary

\section{Tihamer Molnar}

Department of Anaesthesiology and Intensive Care, University of Pecs, Medical School, Pecs, Hungary

\section{Robert Halmosi}

1st Department of Medicine, Division of Cardiology, University of Pecs, Medical School, Pecs, Hungary

\section{Laszlo Deres}

1st Department of Medicine, Division of Cardiology, University of Pecs, Medical School, Pecs, Hungary

\section{Tamas Koszegi}

Department of Laboratory Medicine, University of Pecs, Medical School, Pecs, Hungary

\section{Barbara Sandor}

1st Department of Medicine, Division of Cardiology, University of Pecs, Medical School, Pecs, Hungary Kalman Toth

1st Department of Medicine, Division of Cardiology, University of Pecs, Medical School, Pecs, Hungary Peter Kenyeres ( $\nabla$ kenyeres.peter@pte.hu )

1st Department of Medicine, Division of Cardiology, University of Pecs, Medical School, Pecs, Hungary

\section{Research Article}

Keywords: cardiopulmonary resuscitation, lactate, outcome, post-resuscitation care, cell death, cytokeratin-18 
Posted Date: February 16th, 2021

DOl: https://doi.org/10.21203/rs.3.rs-197502/v1

License: (c) (i) This work is licensed under a Creative Commons Attribution 4.0 International License. Read Full License 


\section{Abstract}

Introduction: We investigated the associations of cell death-related release of cytokeratin-18 (CK-18) and its caspase-cleaved form (CCCK-18) with outcomes, clinical parameters, and neuron-specific enolase (NSE) among cardiac arrest (CA) victims.

Methods: We determined CK-18, CCCK-18, and NSE plasma concentrations within 6, at 24, and 72 post-CA hours of 54 successfully resuscitated patients. Baseline characteristics, laboratory, vital parameters, prognostic scores were recorded. The follow-up was 30 days.

Results: Neither the absolute values of CK-18, CCCK-18, CCCK-18/CK-18 ratio, or NSE, nor their kinetics showed significant difference according to survival and neurological outcome. CK-18 decreased in good renal function in contrast to renal failure. Lactate levels were increased in non-survivors and poor neurological outcomes. In receiver operator characteristic (ROC) analyses lactate was comparable with prognostic scores in predicting 30-day mortality and neurological outcome. The ROC curve of initial lactate indicated a cut-off as $4.90 \mathrm{mmol} / \mathrm{L}$ to predict 30 -day mortality (specificity=80\%; sensitivity=74\%) and $6.00 \mathrm{mmol} / \mathrm{L}$ (specificity $=80 \%$; sensitivity $=84 \%$ ) for poor neurological outcome.

Conclusion: This study was the first to investigate the post-resuscitation prognostic value of CK-18 and CCCK-18 with NSE. Although we could not prove their prognostic value, we confirmed the role of initial lactate in predicting mortality and neurological outcome in unselected resuscitated patients.

\section{Introduction}

Even after successful cardiopulmonary resuscitation (CPR), the long-term survival of cardiac arrest (CA) victims remains poor despite all efforts ${ }^{1,2}$. Early death within 3 days after out-of-hospital cardiac arrest (OHCA) occurs mostly due to circulatory failure, while later death is mainly related to severe hypoxicischemic encephalopathy and the subsequent withdrawal of life-sustaining therapy following prognostication of poor neurological outcome, which is responsible for approximately half of the deaths 3,4. Two-thirds of patients surviving admission to intensive care unit (ICU) after OHCA die due to brain injury, while neurological death only occurs in one-fourth of patients with in-hospital cardiac arrest (IHCA), in whom multiple organ failure drives mortality ${ }^{5,6}$.

Predicting the overall survival and neurological function of CA victims is amongst the biggest challenges facing the medical team. Early predictors of outcome that would support clinical decision-making are required to avoid costly and intensive resources in cases of futility, to guide level-of-care decisions and goals-of-care conversations with family members ${ }^{7}$. The current guidelines and studies are mostly dealing with neurological prognostication in patients remaining comatose after $\mathrm{CA}{ }^{8,9}$, while high proportion of patients (especially after IHCA) reach acceptable neurological function but suffer from multiple organ failure, which may lead to death independently of neurological status ${ }^{10}$. 
In the past decades, multiple biomarkers have been tested for neuroprognostication after CA (e.g. NSE neuron-specific enolase; S100 calcium-binding protein B; neurofilament light chain, tau protein, glial fibrillary acidic protein) ${ }^{11}$, which are released by injury of neuronal or glial cells reflecting the neurological function. Besides, it would be necessary to find a reliable biomarker in unselected resuscitated population after cardiac arrest which could provide useful information about the general outcome and survival without focusing only on the neurological status.

Ischemia and reperfusion cause intracellular $\mathrm{Ca}^{2+}$ overload and generation of reactive oxygen species conditions, what predisposes to mitochondrial injury ${ }^{12}$ and cytochrome $c$ release ${ }^{13}$ resulting in the activation of executioner caspases and cleavage of cytokeratins through complex biochemical pathways 14,15. Cytochrome $\mathrm{c}$ - as a cell death marker - is released into the bloodstream after resuscitation from cardiac arrest in a rat model of ventricular fibrillation and closed-chest resuscitation ${ }^{16}$. Cytokeratins are proteins of the intermediate filament group and components of intracytoplasmic cytoskeleton in the epithelial and parenchymal cells ${ }^{17}$. Ischemia-reperfusion injury as the consequence of CA and CPR leads to increased systemic apoptotic and necrotic cell death ${ }^{18}$. The activity of caspases during apoptosis leads to the fragmentation of the cytokeratin-18 (CK-18), which is considered to be an apoptosis-specific cell death biomarker (caspase-cleaved cytokeratin-18 - CCCK-18), while necrotic cell death results in the release of the full-length CK-18 to the circulation. The fragments of the CK-18 cleaved by caspases can be recognised by a monoclonal antibody and in combination with the full CK-18 measurement, the predominant mode of cell death can be determined ${ }^{19}$.

Previous studies found an association between the increased levels of CK-18 and its caspase cleaved fragments and outcome in different disorders. The increased level of CCCK-18 in septic and critically ill patients was associated with mortality in previous studies ${ }^{20}$. CCCK-18 concentrations are elevated in patients with acute myocardial infarction compared to stable or unstable angina patients and the marker is significantly increased at the site of coronary occlusion as compared to peripheral blood samples ${ }^{21}$. More studies investigated the marker in neurological disorders, such as ischemic stroke ${ }^{22}$, intracerebral ${ }^{23}$, and aneurysmal subarachnoid hemorrhage ${ }^{24}$, and traumatic brain injury ${ }^{25}$.

These considerations suggest that CK-18 and CCCK-18 could provide additional information to the prognosis of CA survivors as well since this condition often leads to critical illness and neurological disorder. We hypothesised that the level of systemic cell death should be in association with the mortality and outcome after CA. Therefore, the following study was performed to characterise for the first time the release pattern of CK-18 and CCCK-18 in a prospective unselected cohort of resuscitated patients to evaluate their prognostic value for mortality, outcome, and their association with previously studied NSE, conventionally used clinical and laboratory parameters, prognostic scoring systems.

\section{Results}

\section{Baseline characteristics}


Characteristics, etiology of the CA and CPR, vital parameters and comorbidities of the study cohort are summarized in Table 1. 54 patients were enrolled (median age: 67 [61-78] years, 48\% male), $72 \%$ suffered IHCA, $69 \%$ had non-shockable initial rhythm. We did not find statistically significant differences between non-survivors $(n=38 ; 70 \%)$ and survivors on age, gender, and the duration of CPR. Among survivors, the CPC category was significantly better, as expected (3.0 [2.3-3.0] vs. 4.5 [3.0-5.0], $p<0.001)$ and significantly favourable SAPS II and SOFA scores were recorded as shown in Table 1. 
Table 1

Characteristics of the study population according to 30-day mortality

\section{Survivors $(n=16$; \\ $30 \%)$ \\ Non-survivors $(n=38$; \\ 70\%) \\ p value}

Baseline

Age (years)

$61[58-76]$

$69[62-80]$

0.134

Male gender

$5(31 \%)$

$21(55 \%)$

0.107

Characteristics of the CA and the CPR

Localisation: in-hospital CA

$13(81 \%)$

$26(68 \%)$

0.337

Resuscitation during nightshift or

$10(63 \%)$

$29(76 \%)$

0.301

weekend

First monitored rhythm:

- Ventricular tachycardia/fibrillation

$4(25 \%)$

$10(26 \%)$

0.920

- Pulseless electrical activity

$3(19 \%)$

$11(29 \%)$

0.435

- Asystolia

$8(50 \%)$

$15(40 \%)$

0.475

- Unknown

$1(6 \%)$

$2(5 \%)$

0.885

Time of the resuscitation ( $\mathrm{min}$ )

10 [5-23]

10 [5-20]

0.916

Patients required epinephrine

$11(69 \%)$

$34(90 \%)$

0.062

Dose of epinephrine (mg)

$2[0-3]$

$2[1-3]$

0.234

Mechanical ventilation within 6 hours

after CA

$15(94 \%)$

$34(90 \%)$

0.621

\section{Etiology of CA}

Ischaemic heart disease

$6(38 \%)$

$11(29 \%)$

0.537

Heart failure

$6(38 \%)$

$10(26 \%)$

0.411

Sepsis

$1(6 \%)$

$4(11 \%)$

0.621

Hyperkalaemia

$1(6 \%)$

$4(11 \%)$

0.621

Aspiration

$1(6 \%)$

2 (5\%)

0.885

Hypothermia

1 (6\%)

1 (3\%)

0.520

Stroke

0

2 (5\%)

0.350

Continuous data are presented as median values with interquartile range [percentiles 25-75] or mean \pm standard deviation, categorical data as the number of subjects and percentages. CA: cardiac arrest, CPR: cardiopulmonary resuscitation; SOFA: Sequential Organ Failure Assessment Score; SAPS II: Simplified Acute Physiology Score II; ICU: intensive care unit. 


\section{$\begin{array}{lll}\text { Survivors }(n=16 ; & \begin{array}{l}\text { Non-survivors }(n=38 ; \\ 30 \%)\end{array} & \begin{array}{l}p \\ \text { value }\end{array}\end{array}$}

\begin{tabular}{|llll|}
\hline Pulmonary embolism & 0 & $2(5 \%)$ & 0.350 \\
\hline Pneumonia & $1(6 \%)$ & $1(3 \%)$ & 0.520 \\
\hline Unknown & $6(38 \%)$ & $11(29 \%)$ & 0.537 \\
\hline Parameters on enrolment & & & \\
\hline Systolic blood pressure $(\mathrm{mmHg})$ & $115[104-133]$ & $114[102-138]$ & 0.896 \\
\hline Diastolic blood pressure $(\mathrm{mmHg})$ & $61[55-69]$ & $61[54-68]$ & 0.905 \\
\hline Mean arterial pressure $(\mathrm{mmHg})$ & $77[71-91]$ & $77[70-86]$ & 0.842 \\
\hline Heart rate $(/$ min) & $76[66-90]$ & $91[70-105]$ & 0.065 \\
\hline Body temperature $\left.{ }^{\circ} \mathrm{C}\right)$ & $36.6 \pm 0.7$ & $36.2 \pm 1.5$ & 0.269 \\
\hline Comorbidities, previous medical history & & & 0.767 \\
\hline Hypertension & $12(75 \%)$ & $27(71 \%)$ & 0.235 \\
\hline Ischaemic heart disease & $4(25 \%)$ & $16(42 \%)$ & 0.127 \\
\hline Diabetes mellitus & $4(25 \%)$ & $18(47 \%)$ & 0.537 \\
\hline Heart failure & $6(38 \%)$ & $11(29 \%)$ & 0.977 \\
\hline Permanent atrial fibrillation & $3(19 \%)$ & $7(18 \%)$ & 0.132 \\
\hline Stroke or transient ischemic attack & $1(6 \%)$ & $9(24 \%)$ & 0.594 \\
\hline Carotid artery stenosis & $2(13 \%)$ & $3(8 \%)$ & 0.027 \\
\hline Chronic obstructive pulmonary disease & $5(31 \%)$ & $3(8 \%)$ & 0.341 \\
\hline Peripheral artery disease & $1(6 \%)$ & $6(16 \%)$ & 0.597 \\
\hline Previous pulmonary embolism & $1(6 \%)$ & $(13 \%)$ & \\
\hline Previous, cured malignant disease & $3(19 \%)$ & & \\
\hline & & & \\
\hline
\end{tabular}

Continuous data are presented as median values with interquartile range [percentiles 25-75] or mean \pm standard deviation, categorical data as the number of subjects and percentages. CA: cardiac arrest, CPR: cardiopulmonary resuscitation; SOFA: Sequential Organ Failure Assessment Score; SAPS II: Simplified Acute Physiology Score II; ICU: intensive care unit. 


\begin{tabular}{|c|c|c|c|}
\hline & $\begin{array}{l}\text { Survivors }(n=16 \\
30 \%)\end{array}$ & $\begin{array}{l}\text { Non-survivors ( } n=38 \\
70 \% \text {; }\end{array}$ & $\begin{array}{l}p \\
\text { value }\end{array}$ \\
\hline Active malignant or hematologic disease & $2(13 \%)$ & $7(18 \%)$ & 0.594 \\
\hline \multicolumn{4}{|l|}{ Prognostic scores } \\
\hline SOFA & $9 \pm 4$ & $11 \pm 3$ & 0.046 \\
\hline SAPS II & $64 \pm 17$ & $80 \pm 14$ & $<.001$ \\
\hline \multicolumn{4}{|c|}{$\begin{array}{l}\text { Continuous data are presented as median values with interquartile range [percentiles } 25-75] \text { or mean } \\
\pm \text { standard deviation, categorical data as the number of subjects and percentages. CA: cardiac arrest, } \\
\text { CPR: cardiopulmonary resuscitation; SOFA: Sequential Organ Failure Assessment Score; SAPS II: } \\
\text { Simplified Acute Physiology Score II; ICU: intensive care unit. }\end{array}$} \\
\hline
\end{tabular}

\section{Investigating biomarkers according to 30-day mortality and neurological outcome}

Box plots in Fig. 1. a-d. show plasma CK-18, CCCK-18, CCCK-18/CK-18 ratio, and NSE values measured at 6,24 , and 72 hours according to 30 -day survival. None of the markers or their kinetics showed significant difference between survivors and non-survivors. The subgroup analysis of IHCA or OHCA survivors and non-survivors did not reveal difference in the cell death marker levels at 6, 24, or 72 hours either (Suppl. Fig. 1.) Neither the initial rhythm nor the length of resuscitation affected the marker levels (Suppl. Fig. 2-3). We could not demonstrate an association between the higher number of organ system failure and enhanced cell death marker levels (Suppl. - Fig. 4.). On the other hand, subgroup analysis revealed that the CK-18 level did not decrease during the investigated time in renal failure compared to normal renal function subgroup. The difference became significant at 72 hours (Fig. 2.). Non-significant results related to other organ failure have not been shown. We could not confirm significant connection between the cell death marker levels and neurological outcome either (Suppl. - Fig. 5.).

\section{Routine laboratory parameters}

We recorded and analysed the main conventionally used laboratory parameters regarding 30-day mortality summarised in Table 2. Non-survivors had elevated initial liver function parameters and troponin. Hemoglobin and hematocrit values were lower among survivors at 24 and 72 post-CA hours as shown in Table 2. White blood cell and neutrophil count were elevated among non-survivors at 72 post-CA hours. Lactate levels were increased among non-survivors at 6-hour (3.2 [2.1-4.6] vs. 7.1 [4.3-9.7]; $p=$ $0.005)$ and 24 -hour (1.0 [0.8-1.5] vs. $1.7[1.1-2.5] ; p=0.008)$ as well and patients with higher initial lactate (3.6 [2.1-5.7] vs. 8.7 [6.0-10.7]; $p<0.001)$ or 24-hour lactate levels (1.1 [0.9-1.7] vs. 1.8 [1.1-2.5]; $p=0.030)$ were more prone to have poor (CPC4-5) neurological status (Fig. 3. $a-b$.). 
Table 2

Routine laboratory parameters and 30-day mortality

$\begin{array}{lll}\text { Survivors }(n=16 ; & \begin{array}{l}\text { Non-survivors }(n=38 ; \\ 30 \%)\end{array} & \begin{array}{l}p \\ \text { value }\end{array}\end{array}$

Laboratory parameters within 6 hours after $\mathrm{CA}$

\begin{tabular}{|c|c|c|c|}
\hline blood urea nitrogen $(\mathrm{mmol} / \mathrm{l})$ & 6.64 [3.99-12.89] & $10.72[7.32-15.32]$ & NS \\
\hline creatinine (umol/I) & $\begin{array}{l}113.00[83.00- \\
256.00]\end{array}$ & $113.00[95.50-186.75]$ & NS \\
\hline lactic dehydrogenase (IU/I) & $\begin{array}{l}421.00[312.25- \\
687.00]\end{array}$ & $\begin{array}{l}1096.00[642.00- \\
2354.50]\end{array}$ & 0.001 \\
\hline $\begin{array}{l}\text { glutamic oxaloacetic transaminase } \\
(\mathrm{IU} / \mathrm{I})\end{array}$ & $27.00[24.00-100.50]$ & $98.50[41.50-680.25]$ & 0.003 \\
\hline glutamic pyruvic transaminase (IU/I) & $18.00[13.00-62.00]$ & $51.00[24.50-582.00]$ & 0.020 \\
\hline total bilirubin (umol/l) & $6.90[4.45-18.15]$ & $13.30[6.25-23.1]$ & NS \\
\hline Troponin-T (ng/mL) & $44.98[27.11-87.05]$ & $142.30[52.61-389.60]$ & 0.013 \\
\hline INR & $1.19[1.03-1.28]$ & $1.35[1.12-1.76]$ & 0.050 \\
\hline CRP (mg/l) & $17.50[2.48-97.20]$ & $55.35[7.60-117.43]$ & NS \\
\hline white blood cell count (G/I) & $12.45[9.50-16.11]$ & $13.95[9.67-21.82]$ & NS \\
\hline neutrophil (G/I) & $9.07[5.94-12.93]$ & $11.50[6.96-15.08]$ & NS \\
\hline hemoglobin ( $g / \mathrm{l})$ & $\begin{array}{l}101.50[92.00- \\
120.00]\end{array}$ & $116.00[97.75-134.50]$ & NS \\
\hline hematocrit (\%) & $32.75[29.15-38.00]$ & $35.85[29.38-42.00]$ & NS \\
\hline platelet count $(G / I)$ & $\begin{array}{l}267.00[179.75- \\
381.25]\end{array}$ & $236.00[174.75-336.75]$ & NS \\
\hline
\end{tabular}

Laboratory parameters 24 hours after CA

blood urea nitrogen $(\mathrm{mmol} / \mathrm{l})$

creatinine (umol/l)

$\operatorname{CRP}(\mathrm{mg} / \mathrm{l})$

white blood cell count $(G / I)$

neutrophil $(G / l)$
$7.03[4.90-10.32] \quad 12.52[7.99-19.32]$

$145.50[89.00-223.00]$

202.50]

$61.75[29.53-90.88]$

$10.19[7.19-19.97]$

$8.23[5.33-18.75]$
$77.45[31.78-129.68]$

$12.42[9.67-19.15]$

$11.12[8.83-17.00]$
0.009

NS

NS

NS

NS

Continuous data is presented as median value with interquartile range (percentiles $25-75$ ), categorical data as number of subjects and percentages. $(C A=$ cardiac arrest; $\mathrm{CRP}=\mathrm{C}$-Reactive Protein; INR = International Normalized Ratio; NS = non-significant $p>0.05$ ) 


\begin{tabular}{|c|c|c|c|}
\hline & $\begin{array}{l}\text { Survivors ( } n=16 \text {; } \\
30 \%)\end{array}$ & $\begin{array}{l}\text { Non-survivors }(n=38 \text {; } \\
70 \%)\end{array}$ & $\begin{array}{l}\mathrm{p} \\
\text { value }\end{array}$ \\
\hline hemoglobin (g/l) & $92.50[82.50-115.50]$ & $115.00[103.50-133.00]$ & 0.015 \\
\hline hematocrit (\%) & $28.35[25.35-37.33]$ & $34.80[30.70-40.20]$ & 0.018 \\
\hline platelet count (G/I) & $\begin{array}{l}237.00[145.75- \\
369.75]\end{array}$ & $267.00[142.50-321.50]$ & NS \\
\hline \multicolumn{4}{|c|}{ Laboratory parameters 72 hours after $\mathrm{CA}$} \\
\hline blood urea nitrogen $(\mathrm{mmol} / \mathrm{l})$ & $6.62[5.02-9.08]$ & $10.68[6.77-17.93]$ & NS \\
\hline creatinine (umol/l) & $86.50[67.00-143.50]$ & $131.00[76.00-237.00]$ & NS \\
\hline CRP (mg/l) & $94.85[50.65-156.05]$ & $136.70[60.20-224.60]$ & NS \\
\hline white blood cell count (G/l) & $9.20[6.82-12.76]$ & $16.27[12.91-20.14]$ & 0.002 \\
\hline neutrophil (G/I) & 7.77 [5.65-11.27] & $13.06[10.34-17.92]$ & 0.002 \\
\hline hemoglobin $(\mathrm{g} / \mathrm{l})$ & $91.00[80.75-112.00]$ & $111.50[94.25-124.00]$ & 0.033 \\
\hline hematocrit (\%) & $27.70[25.50-34.18]$ & $33.35[28.68-39.00]$ & 0.050 \\
\hline platelet count (G/I) & $\begin{array}{l}192.50[109.50- \\
293.25]\end{array}$ & 197.00 [126.00-275.50] & NS \\
\hline
\end{tabular}

Continuous data is presented as median value with interquartile range (percentiles 25-75), categorical data as number of subjects and percentages. (CA = cardiac arrest; $\mathrm{CRP}=\mathrm{C}$-Reactive Protein; INR = International Normalized Ratio; NS = non-significant $p>0.05$ )

\section{Prognostication of 30-day mortality and neurological outcome}

None of the investigated biomarkers (CK-18, CCCK-18, or NSE) or their change were found to be significantly related to 30-day mortality with univariable regression analysis (Suppl. - Table 1.). However, initial lactate seemed to predict 30-day mortality and neurological outcome, while 24 and 72-hour lactate values had less prognostic accuracy, so we considered only initial lactate in the later analysis. ROC analysis for prediction of the 30-day mortality was carried out with SOFA, SAPS II, and lactate levels. AUC values were 0.650 [0.474-0.825] $(p=0.094)$ for SOFA, 0.757 [0.601-0.913] $(p=0.001)$ for SAPS II and 0.745 [0.593-0.897] $(p=0.002)$ for lactate, respectively. In mortality prediction, the SOFA had poor, SAPS II had moderate value, while the AUC of lactate per se was similar to SAPS II based on ROC analysis. There was no statistically significant difference comparing the AUC values (SAPS II vs. lactate $p=0.892$; SAPS II vs. SOFA $p=0.088$; lactate vs. SOFA $p=0.373$ ). (Fig. 4. a.)

ROC analysis was performed with the same variables for prediction of poor neurological outcome (CPC45). AUC values were 0.697 [0.548-0.845] $(p=0.009)$ for SOFA, $0.763[0.626-0.900](p<0.001)$ for SAPS II 
and 0.835 [0.720-0.951] $(p<0.001)$ for lactate. As for poor neurological outcome, lactate per se had good predictive value however, the differences of the AUC values were not statistically significant (SAPS II vs. lactate $p=0.356$; SAPS II vs. SOFA $p=0.322$; lactate vs. SOFA $p=0.129$ ). (Fig. 4. b.)

The ROC curve of initial lactate per se indicated a cut-off as $4.90 \mathrm{mmol} / \mathrm{L}$ to predict 30-day mortality (sensitivity: $74 \%$; specificity: $80 \%$ ) and as $6.00 \mathrm{mmol} / \mathrm{L}$ (specificity: $80 \%$, sensitivity: $84 \%$ ) for poor neurological outcome (CPC4-5).

\section{Discussion}

To date, this is the first study investigating the prognostic value for mortality and neurological outcome of CK-18 and its caspase-cleaved form in an unselected resuscitated population. The levels of the CK-18 referring to total cell death including apoptosis and necrosis were elevated, and the CCCK-18/CK-18 ratio was decreased among resuscitated patients compared to other study populations, that refers to a large extent of cell death dominantly due to necrosis ${ }^{26}$. Contrary to expectations, the investigated biomarkers or their change did not differ based on 30-day mortality. None of these markers showed connection with neurological function either. Despite our initial hypothesis, (i.e. survival depends on the rate of cell death after CA) mortality could rather be determined by damage of a smaller group of cells responsible for critical function and survival, but this signal may vanish in the mass of the total cell death. Survival may also rather depend on the remaining functional capacity and the ability to recover than the extent of damage that the above biomarkers may represent.

More surprisingly, no correlation was found between the biomarkers and the duration of CPR either, though a longer state of arrest is expected to cause a larger extent of cell damage. On the other hand, information about length and severity of prearrest hypoxia, and time between CA and start of effective resuscitation was lacking, though this period may not only be as important as the duration of resuscitation but also interferes with the results. Similarly, no correlation was found between the duration of resuscitation and survival.

We observed similarly negative results when evaluating the relationship between other organ system failure and cell death markers, except renal function. While CK-18 levels show a slowly declining trend over time in patients with normal renal function, it remains high in patients with renal insufficiency. The significantly higher 72-hour CK-18 levels in patients with renal failure after CA mostly could be due to the impaired renal elimination and less probably due to increased release from injured renal epithelial cells 27,28 . In a previous study increased serum concentration of total CK-18 was found in patients with chronic kidney disease stages 3-5, while there was no significant elevation of CCCK-18 levels similar to our findings ${ }^{29}$.

We could not prove any connection of the cell death biomarkers with neurological outcome. Practically the peripheral blood levels of CK-18 and CCCK-18 do not refer to neuronal cell death, as the cytokeratins appear mostly in the epithelial cells but not in the neuronal cells, where the intermediate filaments are

Page $11 / 22$ 
made up of neurofilaments ${ }^{30}$. The source of the elevated levels of cytokeratins in other studies dealing with neurological disorders could be the damage of epithelial cells due to other organ failure or of cells of the perineurial and arachnoidal sheaths ${ }^{31}$.

Consequently, we tested a marker that represents neuronal injury more specifically. In previous studies, NSE was described as a prognostic marker for poor neurological outcome after cardiac arrest and it has an additional role in neuroprognostication in the current guidelines ${ }^{11}$. In contrast to our expectations, there was no significant difference in our study population concerning this marker and we could not confirm the prognostic value for 30-day mortality or neurological outcome. Explanation could be the high heterogeneity of our unselected population, which was mostly composed of various IHCA cases, and even the OHCA group had diverse etiology. Only a few papers published data about the prognostic value of NSE in patients after IHCA ${ }^{32}$. The prognostic value of NSE was higher for OHCA than for IHCA patients in the study of Kaspar et al. ${ }^{33}$. They explained the difference with higher number of confounders and mortality without hypoxic-ischemic encephalopathy in IHCA patients.

Failure of tissue perfusion during cardiac arrest leads to anaerobic metabolism. Lactate is the end product of anaerobic metabolism that can be used as a marker of cellular hypoxia and to predict mortality in critical illness ${ }^{34}$. Previous investigations have proved the utility of lactate as a marker for disturbances of tissue perfusion to predict survival in cardiac arrest patients ${ }^{35,36}$. According to our results, the lactate values per se had moderate prognostic value for 30-day mortality compared to the ones of SOFA and SAPS II. The reliability of these widely used prognostic scoring systems is equivocal because of their moderate discrimination ability and they have been consequently considered as not clinically relevant in post-resuscitation care ${ }^{37}$. In a study made up of OHCA patients treated with therapeutic hypothermia, Acute Physiology And Chronic Health Evaluation II, SAPS II, and OHCA scores had moderate accuracy to distinguish neurological outcomes and mortality, moreover, the SOFA score was found to be a poor predictor in this population ${ }^{38}$. Bisbal et al. reported the SAPS III as less accurate in determining the in-hospital mortality of post-CA patients compared to other general prognostic scores 39. Significant efforts have been made in recent years to develop scoring systems that can be used to estimate the outcome of resuscitated patients ${ }^{40}$, and these often require background information about the patient and about often missing peri-arrest circumstances. Though promising, these scores are not as widely used as the classic general prognostic scores.

It was reported by Grimaldi et al. that lactate levels $<5.1 \mathrm{mmol} / \mathrm{L}$ were associated with favourable outcomes at discharge from the intensive care unit after $\mathrm{CA}^{41}$. Similar to their results in our population the cut-off was $4.90 \mathrm{mmol} / \mathrm{L}$ to predict 30-day mortality and $6.00 \mathrm{mmol} / \mathrm{L}$ for poor neurological prognosis. A most recent publication emphasised the need for development of prognostication tool in $\mathrm{IHCA}^{36}$. They retrospectively investigated the predictive role of the initial lactate levels among patients who suffered IHCA and required mechanical ventilation. According to their results elevated lactate levels were associated with mortality and the AUC indicated moderate ability to predict mortality similar to our results. This study mainly focused on mortality, while we evaluated the prognostic role of lactate levels 
concerning the neurological outcome. According to our results elevated lactate levels measured within 6 hours after CA could help the prediction of coma, vegetative state, or brain death.

Although in our recent study we could not prove the prognostic value of novel cell death markers (CCCK18, CK-18), and previously well-investigated NSE, we confirmed the role of initial lactate levels for prediction of mortality and neurological outcome in unselected resuscitated patients. At a quick glance, the simply obtainable early lactate level may give similarly useful information per se than the SAPS II and SOFA scores, which can be calculated later after admission and requires more time.

The strength of our study was the prospective design with an unselected CA patient cohort as we aimed to find a reliable independent biomarker predictor that can be used in general resuscitated population. We performed repeated sampling three times within the first 72 hours after CA instead of only admission sampling to evaluate the change of the investigated biomarkers. The biomarker results (including NSE) were not available to clinicians during patient care and thus could not influence treatment decisions. A major limitation of the current study is the relatively small sample size. The follow-up period ended on the 30 th day after $\mathrm{CA}$, and we have no data about long-term survival or neurological status. Prognostication of neurological outcome after cardiac arrest is based on clinical neurological examination, electrophysiological investigations, neuroimaging, and biomarkers. ${ }^{9}$ However, in our study, we only focused on biomarkers in the peripheral blood and we did not evaluate the mentioned examinations or tools for prognostication. Most of our patients obtained the best CPC of 3, 4, or 5, which are mentioned as poor neurological outcomes in most recent studies contrary to our categorisation (CPC 4-5 for poor and 1-3 for good outcome). Given that the follow-up period was relatively short, we assumed that the fate of patients in CPC 3 category is not hopeless, thus we chose the other classification method.

\section{Conclusion}

In this study we could not prove the prognostic value of the CK-18 and its caspase cleaved form as biomarkers of systemic epithelial cell death, nor the one of NSE, while initial lactate levels were found to be a simple yet moderate predictor for mortality or neurological outcome in our unselected general resuscitated population.

\section{Methods}

\section{Study population}

Adult patients (age $>18$ years) who suffered in-hospital or out-of-hospital CA were prospectively enrolled after successful cardiopulmonary resuscitation to this observational pilot study conducted at the University of Pécs, $1^{\text {st }}$ Department of Medicine, Intensive care unit, at the Department of Anesthesiology and Intensive Care and at the Department of Emergency Medicine from January 2018 to January 2019. Patients with primary traumatic etiology were excluded. Therapeutic hypothermia was not applied however, the overall goal was to maintain normothermia and to prevent fever during post-resuscitation 
care. The study was approved by the Local Ethics Committee of the University of Pécs (file number: 6941 - PTE 2018.) and followed the principles of the Declaration of Helsinki for all human investigations. Before enrolment in the study, written informed consent was obtained from legal representatives or from the alert, oriented patients themselves.

\section{Sample and data collection}

Data collected included patients' anamnestic information, comorbid conditions, variables that are necessary for calculating prognostic scores (Sequential Organ Failure Assessment score - SOFA; Simplified Acute Physiology Score - SAPS II), the circumstances, and presumed cause of the CA, and CPR. We recorded the laboratory parameters (electrolytes; markers of renal and hepatic function; inflammatory parameters; complete blood count, lactate as a marker of anaerobic shift and severe tissue hypoperfusion), blood gas, and vital parameters (heart rate, systolic and diastolic blood pressure, mean arterial pressure, ventilation mode) at the time of blood sampling. We examined the presence of vital organ failure during the post-resuscitation period. Organ system failure was defined as follows:

- circulation: the patient still required vasopressor or inotropic support at 24 hours

- respiration: the patient still required positive pressure ventilation support at 24 hours

- liver: transaminase elevations exceeding three times the normal value

- kidney: decreased urine production ( $<500 \mathrm{ml} /$ day) or creatinine clearance below $30 \mathrm{ml} / \mathrm{min}$.

The SAPS II and SOFA scores were calculated by addition of the worst parameters of the first 24 hours after CA. To avoid false pessimistic estimation of neurological function and to get information about patients who died in ICU or before 30 days, the best neurological status was recorded during ICU stay using the cerebral performance category (CPC) score ${ }^{10,42}$ (Suppl. - Table 2.). We dichotomised patients according to good - CPC 1-3 and poor - CPC 4-5 neurological outcome. The follow-up period was 30 days after $\mathrm{CA}$.

\section{Measurements}

Blood samples were drawn into Vacutainer ${ }^{\circledR}$ EDTA-tubes (Becton Dickinson) within 6 , at $24 \pm 3$ and $72 \pm 3$ hours after CA to determine plasma concentrations of CK-18, CCCK-18, and NSE. The samples were centrifuged within 10 minutes at $1500 \mathrm{~g}$ for 15 minutes. The supernatant was immediately stored in aliquots at $-80^{\circ} \mathrm{C}$ until assayed. CCCK- $18, \mathrm{CK}-18$ and NSE concentrations were determined by using enzyme-linked immunosorbent assay kit (CCCK-18, CK-18 - Shanghai YL Biotech Co., Ltd., China; NSE FineTest, Wuhan Fine Biotech Co., Ltd., China) with the detection limit of $5.64 \mathrm{ng} / \mathrm{L}, 19.00 \mathrm{ng} / \mathrm{L}$ and 1.41 $\mathrm{ng} / \mathrm{mL}$, respectively. We calculated the change of the investigated markers from 6 to 24 and 24 to 72 post-CA hours. The concentration of CK-18 refers to total cell death, while CCCK-18 to apoptosis, since the 
antibody binds only the molecules cleaved by caspases. CCCK-18/CK-18 ratio was calculated at each time point to establish the dominant mode of cell death. All samples were processed by the same technicians using the same equipment and blinded to all clinical data. The biomarker values were blinded to clinicians to avoid the influence on post-resuscitation care approaches or decision-making processes.

\section{Statistical analysis}

Normality of the data was assessed using the Kolmogorov-Smirnov test. Normally distributed continuous variables are expressed as mean and standard deviation, non-normally distributed ones as medians and interquartile ranges, while categorical variables are indicated as frequencies and percentages. MannWhitney U-test was carried out to compare continuous non-normally distributed data between two independent patient groups, while for the statistical analysis of normally distributed variables Student Ttest was applied. Categorical data were compared using the $X^{2}$ test. Univariable binary logistic regression tests were used to evaluate associations between the recorded variables and 30-day mortality with corresponding beta values and $95 \%$ confidence intervals. The diagnostic power of the prognostic scores and lactate was assessed using the area under the curve (AUC) of the receiver operator characteristic (ROC) curve and " $\mathrm{z}$ " test was used for comparison. An AUC value of 0.9 - 1.0 indicates excellent, 0.8 - 0.89 good, 0.7 - 0.79 moderate, 0.6 - 0.69 poor and 0.5 - 0.59 not useful predictive power. The cut-off values of lactate to determine the 30-day mortality and unfavourable neurological outcome was calculated by the ROC analysis. A power analysis was performed for the overall population using PS program version 3.1.2. For the sample size of $n=54$ patients needed to detect a true difference of $\delta=3.42$ in initial lactate according to 30 -day mortality with $84.06 \%$ power, and $\delta=4.09$ in initial lactate according to neurological outcome (CPC 1-3 vs. $4-5$ ) with $95.76 \%$ power, where type I error probability is $a=0.05$. IBM SPSS Statistics ${ }^{\circledR} 27.0$ software was used for statistical analysis of the collected data. A p value $<0.05$ was considered statistically significant.

\section{Declarations}

\section{Acknowledgments}

The authors would like to thank the support and cooperation of the employees of the Intensive care unit of $1^{\text {st }}$ Department of Medicine, the Department of Anaesthesiology and Intensive Care and the Department of Emergency Medicine of the University of Pécs.

\section{Author contributions}

P.K., Z.M., P.C., K.T., R.H. and B.C. designed the study protocol. Z.M., J.R., A.N. and B.C. enrolled the patients, collected the data and samples. T.K., L.D. provided laboratory background, contributed important reagents and performed the measurements. B.C., P.K., T.M. and B.S. analysed the data. B.C., P.K., Z.M. and 
T.M. interpreted the results, wrote the paper. All authors reviewed and approved the paper prior to submission.

\section{Funding}

This work was funded by GINOP 2.3.2.-15-2016-00048. The research was financed by NKFIH in Hungary, within the framework of the 2020-4.1.1-TKP2020 $1^{\text {st }}$ thematic program of the University of Pecs. This work was supported by EFOP-3.6.3- VEKOP-16-2017-00009 at the University of Pecs.

\section{Competing interests}

The authors have no financial involvement with any organization or entity with a financial interest in or financial conflict with the subject matter or materials discussed in the manuscript apart from those disclosed. The authors declare no competing interests.

\section{References}

1. Yan, S. et al. The global survival rate among adult out-of-hospital cardiac arrest patients who received cardiopulmonary resuscitation: a systematic review and meta-analysis. Crit Care.24, 61 https://doi.org/10.1186/s13054-020-2773-2 (2020).

2. Schluep, M., Gravesteijn, B. Y., Stolker, R. J., Endeman, H. \& Hoeks, S. E. One-year survival after inhospital cardiac arrest: A systematic review and meta-analysis. Resuscitation.132, 90-100 https://doi.org/10.1016/j.resuscitation.2018.09.001 (2018).

3. Dragancea, I., Rundgren, M., Englund, E., Friberg, H. \& Cronberg, T. The influence of induced hypothermia and delayed prognostication on the mode of death after cardiac arrest. Resuscitation.84, 337-342 https://doi.org/10.1016/j.resuscitation.2012.09.015 (2013).

4. Dragancea, I. et al. Protocol-driven neurological prognostication and withdrawal of life-sustaining therapy after cardiac arrest and targeted temperature management. Resuscitation.117, 50-57 https://doi.org/10.1016/j.resuscitation.2017.05.014 (2017).

5. Laver, S., Farrow, C., Turner, D. \& Nolan, J. Mode of death after admission to an intensive care unit following cardiac arrest. Intensive Care Med.30, 2126-2128 https://doi.org/10.1007/s00134-0042425-z (2004).

6. Lemiale, V. et al. Intensive care unit mortality after cardiac arrest: the relative contribution of shock and brain injury in a large cohort. Intensive Care Med.39, 1972-1980 https://doi.org/10.1007/s00134-013-3043-4 (2013).

7. Matthews, E. A. et al. Categorization of survival and death after cardiac arrest. Resuscitation.114, 79-82 https://doi.org/10.1016/j.resuscitation.2017.03.005 (2017). 
8. Sandroni, C., Combes, A. \& Nolan, J. P. Focus on post-resuscitation care. Intensive Care Med.45, 1283-1287 https://doi.org/10.1007/s00134-019-05666-2 (2019).

9. Moseby-Knappe, M. et al. Performance of a guideline-recommended algorithm for prognostication of poor neurological outcome after cardiac arrest. Intensive Care Med.46, 1852-1862 https://doi.org/10.1007/s00134-020-06080-9 (2020).

10. Taccone, F. S. et al. Death after awakening from post-anoxic coma: the "Best CPC" project. Crit Care.23, 107 https://doi.org/10.1186/s13054-019-2405-x (2019).

11. Panchal, A. R. et al. Part 3: Adult Basic and Advanced Life Support: 2020 American Heart Association Guidelines for Cardiopulmonary Resuscitation and Emergency Cardiovascular Care. Circulation.142, S366-s468 https://doi.org/10.1161/cir.0000000000000916 (2020).

12. Pittas, K. et al. The Role of Calcium Handling Mechanisms in Reperfusion Injury. Curr Pharm Des.24, 4077-4089 https://doi.org/10.2174/1381612825666181120155953 (2018).

13. de Moissac, D., Gurevich, R. M., Zheng, H., Singal, P. K. \& Kirshenbaum, L. A. Caspase activation and mitochondrial cytochrome $\mathrm{C}$ release during hypoxia-mediated apoptosis of adult ventricular myocytes. J Mol Cell Cardiol.32, 53-63 https://doi.org/10.1006/jmcc.1999.1057 (2000).

14. Caulín, C., Salvesen, G. S. \& Oshima, R. G. Caspase cleavage of keratin 18 and reorganization of intermediate filaments during epithelial cell apoptosis. J Cell Biol.138, 1379-1394 https://doi.org/10.1083/jcb.138.6.1379 (1997).

15. Budihardjo, I., Oliver, H., Lutter, M., Luo, X. \& Wang, X. Biochemical pathways of caspase activation during apoptosis. Annu Rev Cell Dev Biol.15, 269-290 https://doi.org/10.1146/annurev.cellbio.15.1.269 (1999).

16. Radhakrishnan, J. et al. Circulating levels of cytochrome $\mathrm{c}$ after resuscitation from cardiac arrest: a marker of mitochondrial injury and predictor of survival. Am J Physiol Heart Circ Physiol.292, H767775 https://doi.org/10.1152/ajpheart.00468.2006 (2007).

17. Chu, P. G. \& Weiss, L. M. Keratin expression in human tissues and neoplasms. Histopathology.40, 403-439 https://doi.org/10.1046/j.1365-2559.2002.01387.x (2002).

18. Gornik, l. et al. Prognostic value of cell-free DNA in plasma of out-of-hospital cardiac arrest survivors at ICU admission and 24h post-admission. Resuscitation.85, 233-237 https://doi.org/10.1016/j.resuscitation.2013.10.008 (2014).

19. Kramer, G. et al. Differentiation between cell death modes using measurements of different soluble forms of extracellular cytokeratin 18. Cancer Res.64, 1751-1756 https://doi.org/10.1158/00085472.can-03-2455 (2004).

20. Koch, A. et al. High Circulating Caspase-Cleaved Keratin 18 Fragments (M30) Indicate Short-Term Mortality in Critically III Patients. Dis Markers 2018, 8583121, doi:10.1155/2018/8583121 (2018).

21. Adlbrecht, C. et al. Elevated levels of interleukin-1 beta-converting enzyme and caspase-cleaved cytokeratin-18 in acute myocardial infarction. Eur J Clin Invest.37, 372-380 https://doi.org/10.1111/j.1365-2362.2007.01803.x (2007). 
22. Molnar, T. et al. Subacute Elevation of Plasma Level of Caspase-Cleaved Cytokeratin-18 is Associated with Hemorrhagic Transformation and Functional Outcome in Ischemic Stroke. J Stroke Cerebrovasc Dis.28, 719-727 https://doi.org/10.1016/j.jstrokecerebrovasdis.2018.11.015 (2019).

23. Lorente, L. et al. Association between serum levels of caspase-cleaved cytokeratin-18 and early mortality in patients with severe spontaneous intracerebral hemorrhage. BMC Neurosci.19, 23 https://doi.org/10.1186/s12868-018-0424-1 (2018).

24. Yuan, Z. G. et al. Serum caspase-cleaved cytokeratin-18 levels and outcomes after aneurysmal subarachnoid hemorrhage. J Neurol Sci.359, 298-304 https://doi.org/10.1016/j.jns.2015.11.020 (2015).

25. Lorente, L. et al. High Serum Caspase-Cleaved Cytokeratin-18 Levels and Mortality of Traumatic Brain Injury Patients. Brain Sci.9, https://doi.org/10.3390/brainsci9100269 (2019).

26. Hofer, S. et al. Cell death serum biomarkers are early predictors for survival in severe septic patients with hepatic dysfunction. Crit Care.13, R93 https://doi.org/10.1186/cc7923 (2009).

27. Djudjaj, S. et al. Keratins are novel markers of renal epithelial cell injury. Kidney Int.89, 792-808 https://doi.org/10.1016/j.kint.2015.10.015 (2016).

28. De Rosa, S., Antonelli, M. \& Ronco, C. Hypothermia and kidney: a focus on ischaemia-reperfusion injury. Nephrol Dial Transplant.32, 241-247 https://doi.org/10.1093/ndt/gfw038 (2017).

29. Roth, G. A. et al. Increased total cytokeratin-18 serum and urine levels in chronic kidney disease. Clin Chim Acta.412, 713-717 https://doi.org/10.1016/j.cca.2010.12.030 (2011).

30. Kasper, M. Cytokeratins in intracranial and intraspinal tissues. Adv Anat Embryol Cell Biol.126, 1-82 https://doi.org/10.1007/978-3-642-77298-6 (1992).

31. Achtstätter, T., Fouquet, B., Rungger-Brändle, E. \& Franke, W. W. Cytokeratin filaments and desmosomes in the epithelioid cells of the perineurial and arachnoidal sheaths of some vertebrate species. Differentiation.40, 129-149 https://doi.org/10.1111/j.1432-0436.1989.tb00822.x (1989).

32. Rech, T. H., Vieira, S. R., Nagel, F., Brauner, J. S. \& Scalco, R. Serum neuron-specific enolase as early predictor of outcome after in-hospital cardiac arrest: a cohort study. Crit Care.10, R133 https://doi.org/10.1186/cc5046 (2006).

33. Streitberger, K. J. et al. Neuron-Specific Enolase Predicts Poor Outcome After Cardiac Arrest and Targeted Temperature Management: A Multicenter Study on 1,053 Patients. Crit Care Med.45, 11451151 https://doi.org/10.1097/ccm.0000000000002335 (2017).

34. Kruse, O., Grunnet, N. \& Barfod, C. Blood lactate as a predictor for in-hospital mortality in patients admitted acutely to hospital: a systematic review. Scand J Trauma Resusc Emerg Med.19, 74 https://doi.org/10.1186/1757-7241-19-74 (2011).

35. Dadeh, A. A. \& Nuanjaroan, B. Using initial serum lactate level in the emergency department to predict the sustained return of spontaneous circulation in nontraumatic out-of-hospital cardiac arrest patients. Open Access Emerg Med.10, 105-111 https://doi.org/10.2147/oaem.S165154 (2018).

36. Issa, M. S. et al. Lactate and hypotension as predictors of mortality after in-hospital cardiac arrest. Resuscitation.https://doi.org/10.1016/j.resuscitation.2020.10.018 (2020). 
37. Salciccioli, J. D. et al. Performance of SAPS II and SAPS III scores in post-cardiac arrest. Minerva Anestesiol.78, 1341-1347 (2012).

38. Choi, J. Y. et al. Performance on the APACHE II, SAPS II, SOFA and the OHCA score of post-cardiac arrest patients treated with therapeutic hypothermia. PLoS One.13, e0196197 https://doi.org/10.1371/journal.pone.0196197 (2018).

39. Bisbal, M. et al. Effectiveness of SAPS III to predict hospital mortality for post-cardiac arrest patients. Resuscitation.85, 939-944 https://doi.org/10.1016/j.resuscitation.2014.03.302 (2014).

40. Carrick, R. T. et al. Clinical Predictive Models of Sudden Cardiac Arrest: A Survey of the Current Science and Analysis of Model Performances. J Am Heart Assoc.9, e017625 https://doi.org/10.1161/jaha.119.017625 (2020).

41. Grimaldi, D. et al. Short- and long-term outcome in elderly patients after out-of-hospital cardiac arrest: a cohort study. Crit Care Med.42, 2350-2357 https://doi.org/10.1097/ccm.0000000000000512 (2014).

42. Jennett, B. \& Bond, M. Assessment of outcome after severe brain damage. Lancet.1, 480-484 https://doi.org/10.1016/s0140-6736(75)92830-5 (1975).

\section{Figures}


1. a.

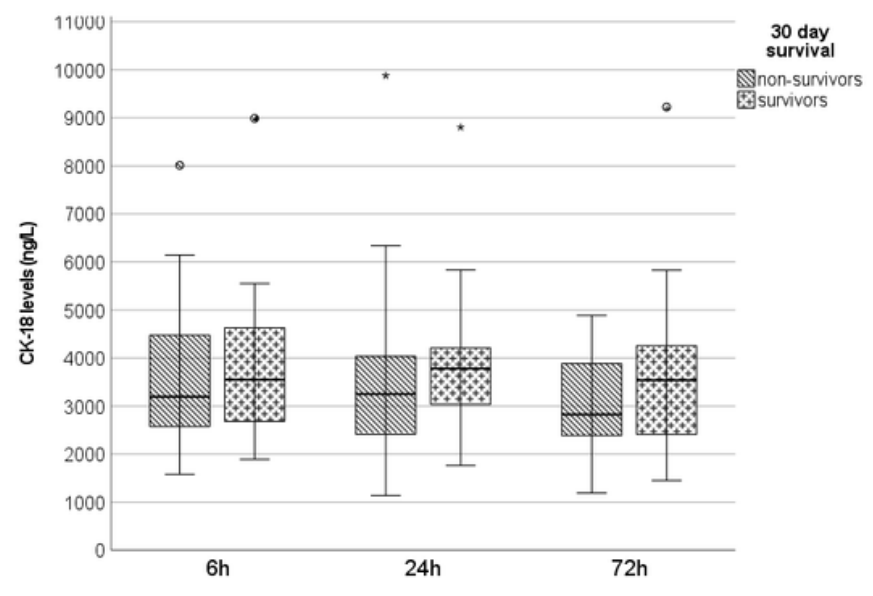

1. c.

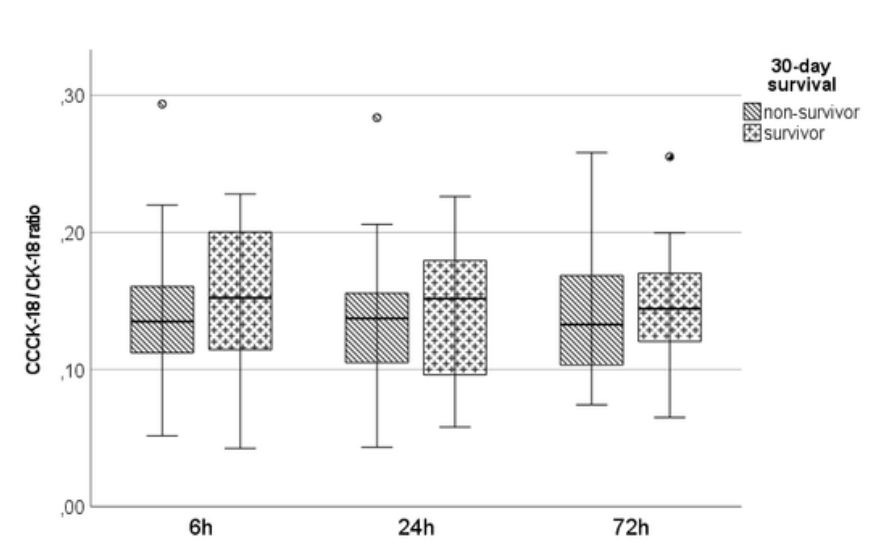

1. b.

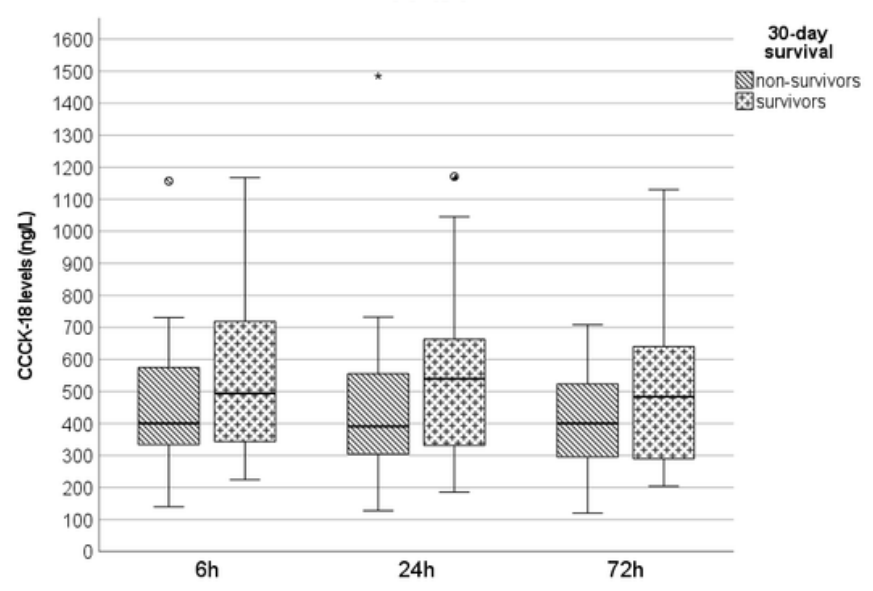

1. d.

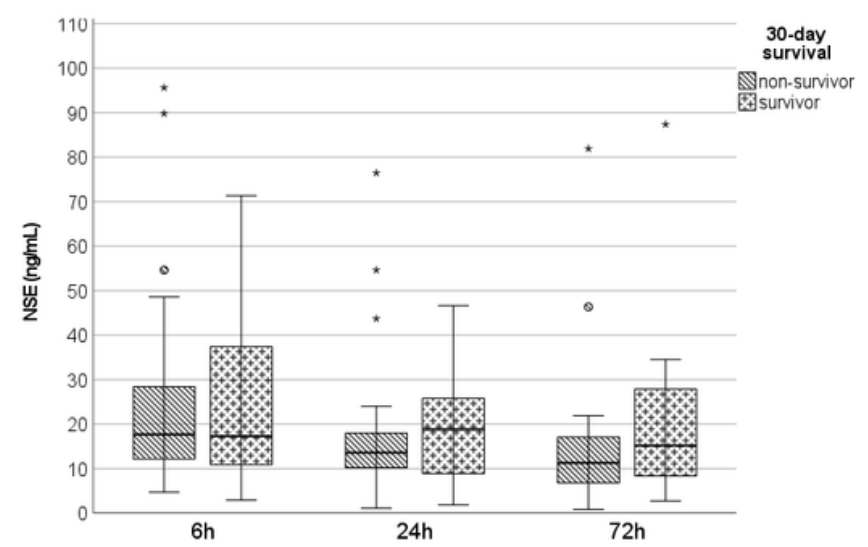

Figure 1

a-d. Box plot diagrams of the CK-18 (1.a.), CCCK-18 (1.b.), CCCK-18/CK-18 ratio (1.c.) and NSE (1.d.) concentrations and within 6 , at 24 and 72 post-cardiac arrest hours according to 30 -day mortality (CK18=cytokeratin-18; CCCK-18=caspase-cleaved cytokeratin-18; NSE=neuron-specific enolase) 


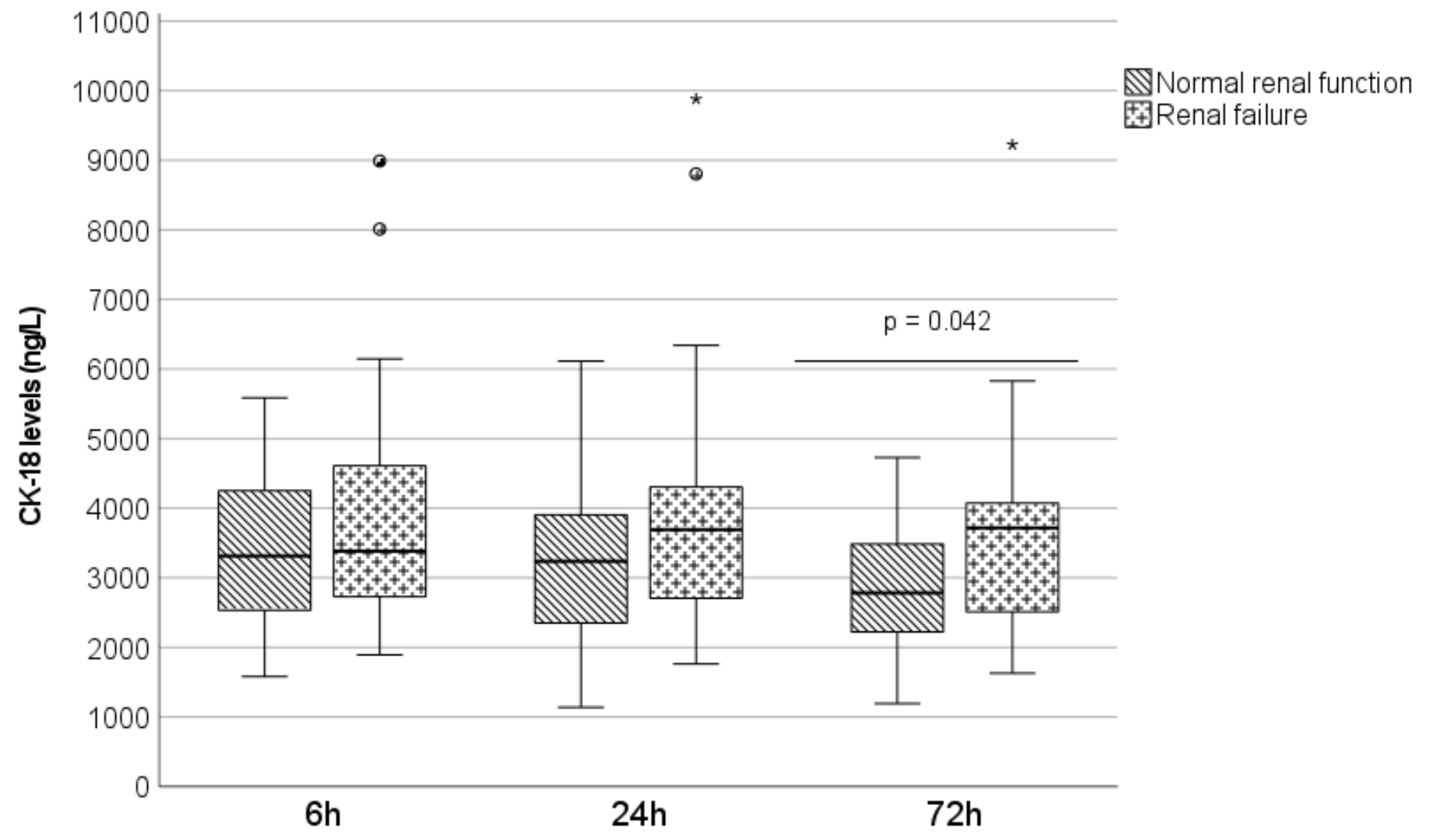

Figure 2

CK-18 levels within 6, at 24 and 72 post-cardiac arrest hours according to renal function (CK18=cytokeratin $-18 ;$ CCCK-18=caspase-cleaved cytokeratin -18 )

3. a.

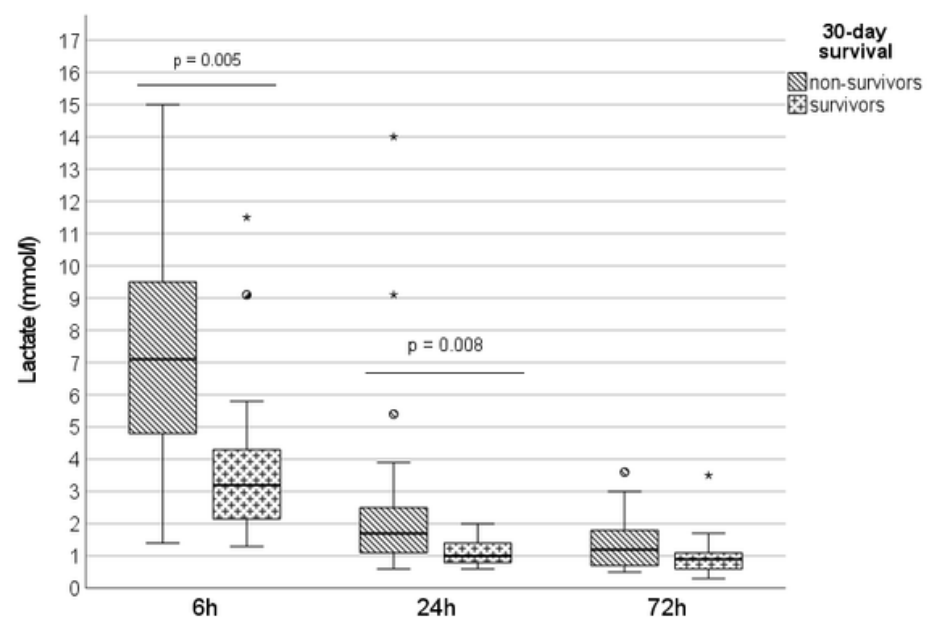

3. b.

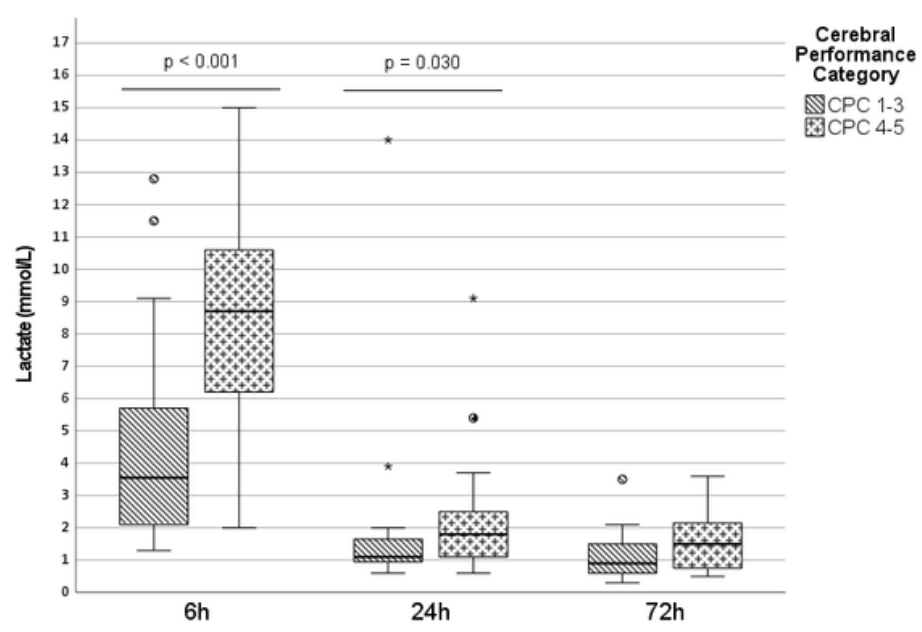

Figure 3 
a-b. Distribution of lactate levels at 6, 24 and 72 hours according to 30-day mortality (3. a.) and neurological outcome (3. b.) (CPC=Cerebral Performance Cetegory)

4. a.

ROC Curve

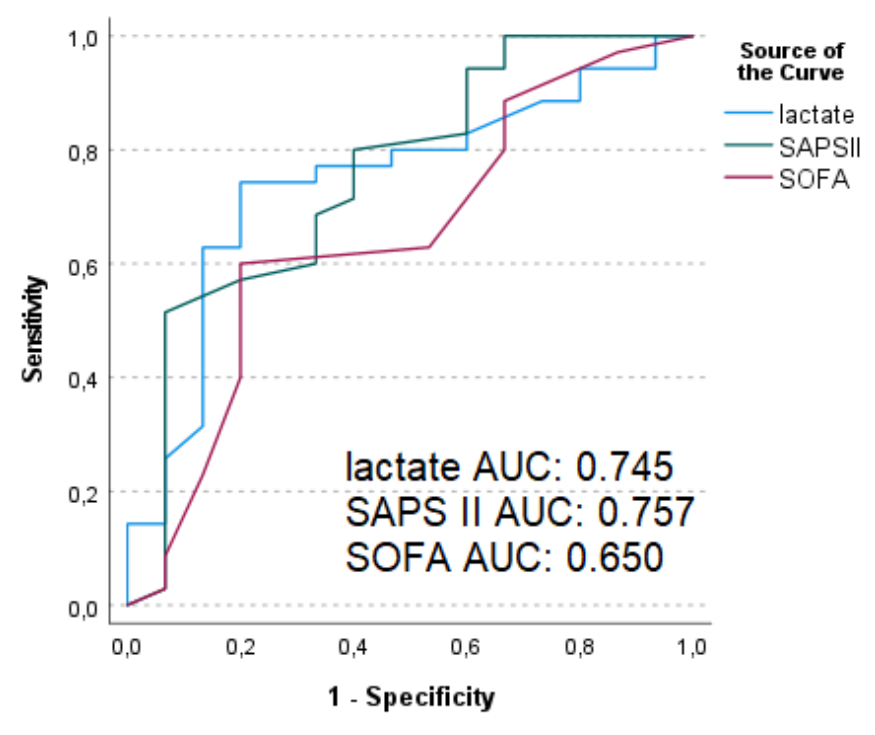

4. b.

Roc Curve

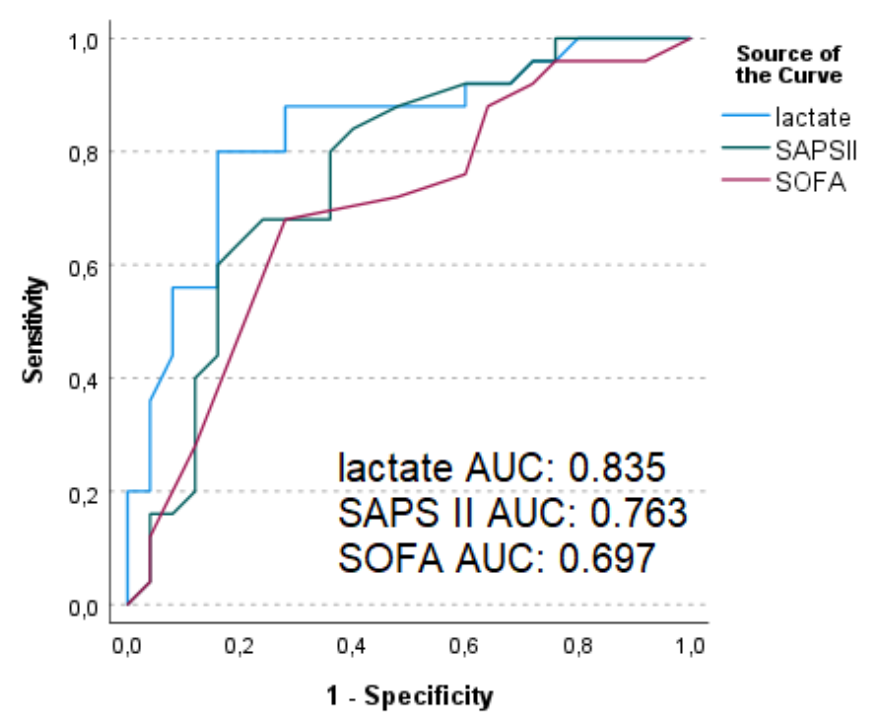

\section{Figure 4}

a-b. ROC Curve of initial lactate, SAPS II and SOFA for 30-day mortality (4. a.) and for poor neurologic outcome (CPC4-5) (4. b.) (AUC= area under the curve; $\mathrm{CPC}=$ Cerebral Performance Category; ROC=receiver operator characteristic; SAPS II=Simplified Acute Physiology Score; SOFA=Sequential Organ Failure Assessment)

\section{Supplementary Files}

This is a list of supplementary files associated with this preprint. Click to download.

- supplementmaterial.docx 